Supporting Information Available: SIO has temperature dependent absorption changes of BR. Temporal analysis and the rationale behind estimation of average life times of BR, GR and TR are also presented. This material is available free of charge via the Internet at http://pubs.acs.org

\title{
Temperature independence of ultrafast photo- isomerization in thermophilic rhodopsin: Assessment vs other microbial proton pumps
}

E. Siva Subramaniam lyer ${ }^{1}$, Ramprasad Misra ${ }^{2}$, Arnab Maity ${ }^{1}$, Oleg Liubashevski ${ }^{1}$, Yuki Sudo $^{3}$, Mordechai Sheves ${ }^{2}$, Sanford Ruhman ${ }^{1 *}$.

${ }^{1}$ Institute of Chemistry, The Hebrew University of Jerusalem, Jerusalem, Israel.

${ }^{2}$ Department of Organic Chemistry, The Weizmann Institute of Science, Rehovot, Israel.

${ }^{3}$ Division of Pharmaceutical sciences, Okayama University, Kita-Ku, Okayama, Japan

Email: sandy@mail.huji.ac.il

Table of Contents

Figure $\mathrm{S} 1$ : Steady State Absorption spectrum of $\mathrm{BR}(\mathrm{pH}=8)$ at different temperatures in the range $20-80{ }^{\circ} \mathrm{C}$

Figure S2. Average lifetime of BR, TR and GR

Figure S3: Bi-exponential fits to the kinetics of stimulated emission in TR, BR, and GR from top to bottom

Figure S4: Species associated spectra for TR

Figure S5: The amplitudes from FFT results of torsional coherences at $20{ }^{\circ} \mathrm{C}$ and $70{ }^{\circ} \mathrm{C}$ for TR.

Derivation for average lifetimes 


\section{Supporting Information}

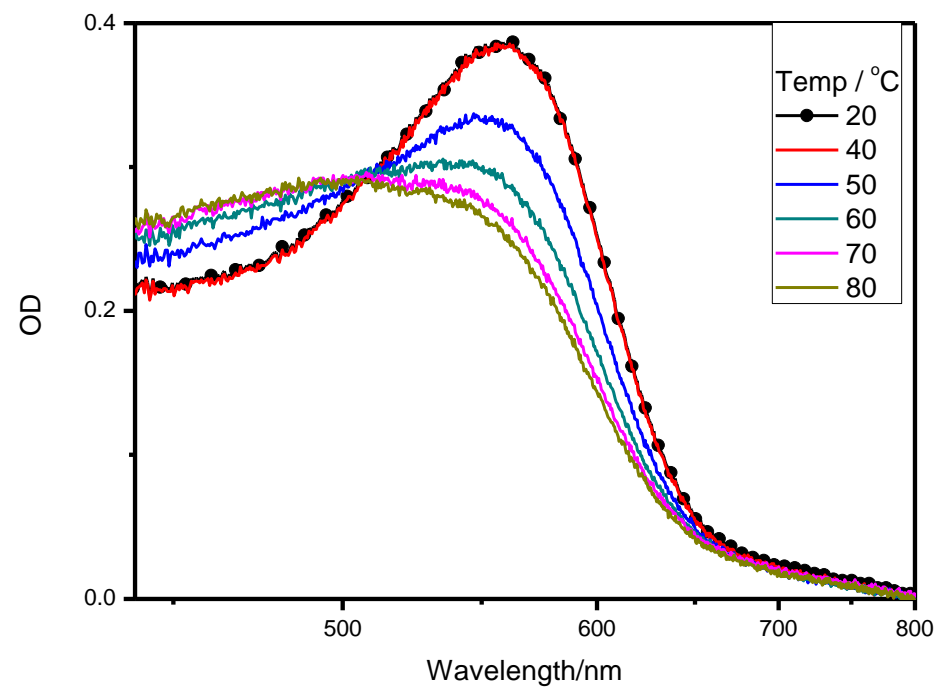

Figure S1: Steady State Absorption spectrum of $\mathrm{BR}(\mathrm{pH}=8)$ at different temperatures in the range $20-80{ }^{\circ} \mathrm{C}$.

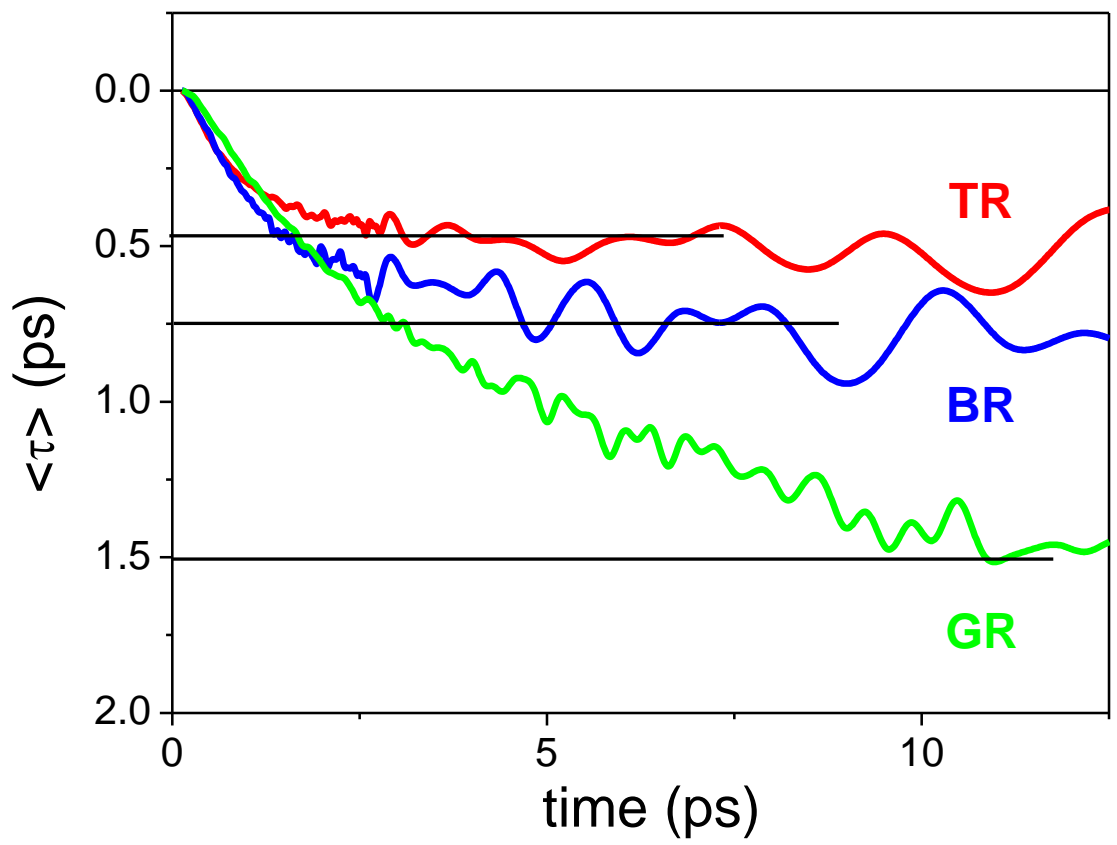

Figure S2. Average lifetime of BR, TR and GR. 
S3
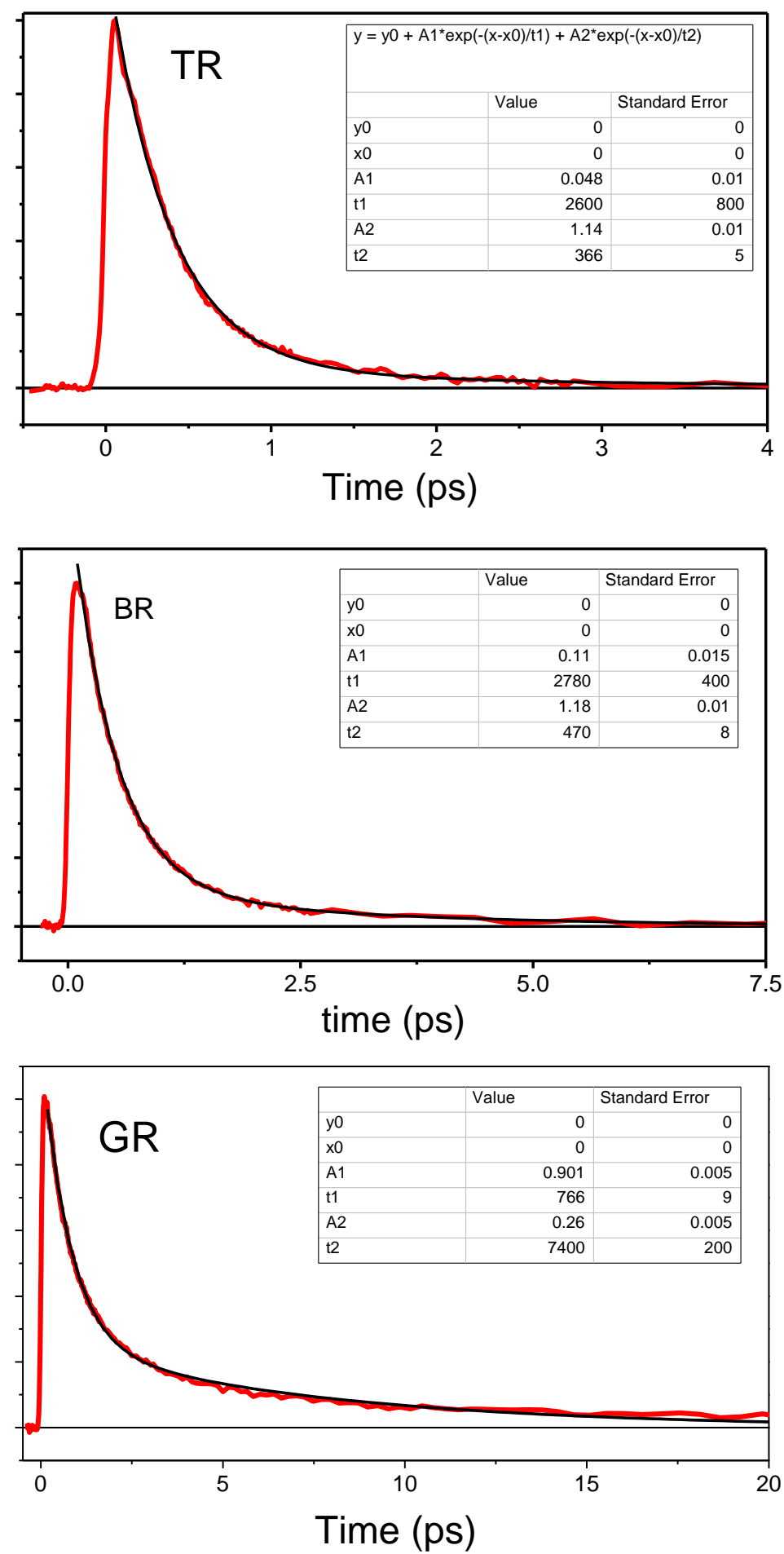

Figure S3: Bi-exponential fits to the kinetics of stimulated emission in TR, BR, and GR from top to bottom. 


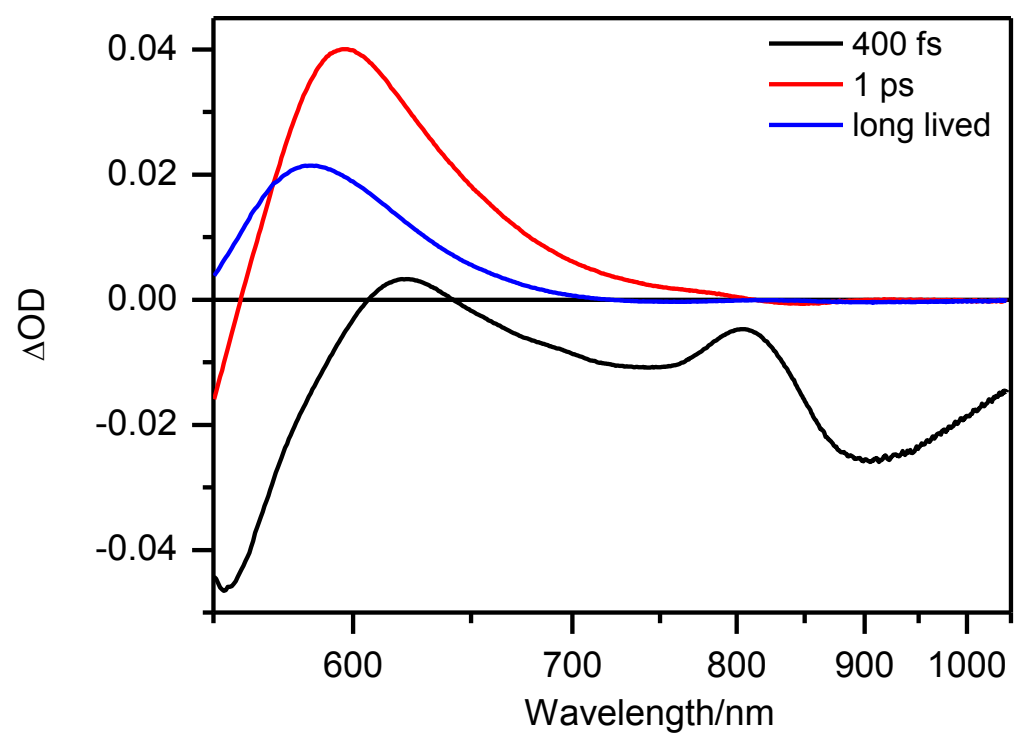

Figure S4: Species associated spectra for TR.

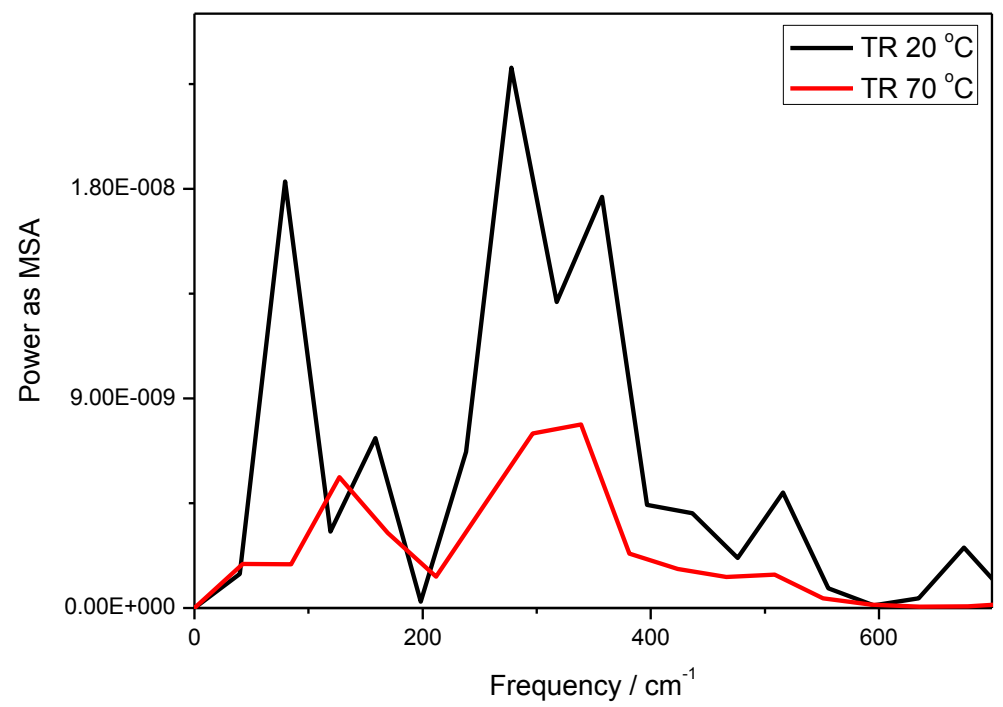

Figure S5: The amplitudes from FFT results of torsional coherences at $20^{\circ} \mathrm{C}$ and $70{ }^{\circ} \mathrm{C}$ for TR. 
Derivation for average lifetimes

Derivation of $<\tau>=\int_{0}^{\infty}\left(\frac{d S_{\text {normalised }}}{d t}\right) t d t$

$S_{\text {normalised }}(t)$ is the normalized monotonic decay of excited state stimulated emission, tracing the time dependence of the probability of an excited protein to still populate $\mathrm{S} 1, t$ femtoseconds after photo-excitation. $\left[S_{\text {normalised }}\left(t^{\prime}+d t\right)-S_{\text {normalised }}\left(t^{\prime}\right)\right]$ is the probability for a protein to decay in the interval from $\left(t^{\prime}\right)$ to $\left(t^{\prime}+d t\right)$, or more simply; $\left[\frac{S_{\text {normalised }}\left(t^{\prime}+d t\right)-S_{\text {normalised }}\left(t^{\prime}\right)}{d t}\right] \equiv \rho(t)$ defines the probability density describing excited state decay. This naturally leads to the equation employed in the paper; $<\tau>\equiv \int_{0}^{\infty} \rho(t) \mathrm{t} d t=\int_{0}^{\infty}\left(\frac{d S_{\text {normalised }}}{d t}\right) t d t$

For example, in the particular case of mono-exponential decay, $S_{\text {normalised }}(t)=e^{-t / \tau} ;\left(\frac{d S_{\text {normalised }}}{d t}\right)=\frac{-1}{\tau} e^{-t / \tau}$
$<\tau>=\int_{0}^{\infty}\left(\frac{d S_{\text {normalised }}}{d t}\right) t d t=\frac{-1}{\tau} \int_{0}^{\infty} t e^{-t / \tau} d t={ }_{0}^{\infty}\left[(t-\tau) e^{-t / \tau}\right]=\tau$

As expected. 